\title{
A Dual-Wavelength Radar Technique to Detect Hydrometeor Phases
}

\author{
Liang Liao ${ }^{1}$ and Robert Meneghini ${ }^{2}$ \\ ${ }^{1}$ Goddard Earth Science Technology \& Research, Morgan State University, MD \\ ${ }^{2}$ NASA Goddard Space Flight Center, Greenbelt, MD
}

Submitted to

IEEE Transaction on Geoscience and Remote Sensing (TGRS)

Corresponding author information:

Dr. Liang Liao

Goddard Earth Sciences Technology and Research

Morgan State University

Code 612

NASA/Goddard Space Flight Center

Greenbelt, MD 20771

301-614-5718 (phone)

301-614-5492 (fax)

Email: Liang.Liao-1@nasa.gov 


\begin{abstract}
This study is aimed at investigating the feasibility of a Ku- and Ka-band space/air-borne dualwavelength radar algorithm to discriminate various phase states of precipitating hydrometeors. A phase-state classification algorithm has been developed from the radar measurements of snow, mixed-phase and rain obtained from stratiform storms. The algorithm, presented in the form of the look-up table that links the Ku-band radar reflectivities and dual-frequency ratio (DFR) to the phase states of hydrometeors, is checked by applying it to the measurements of the Jet Propulsion Laboratory, California Institute of Technology, Airborne Precipitation Radar Second Generation (APR-2). In creating the statistically-based phase look-up table, the attenuationcorrected (or true) radar reflectivity factors are employed, leading to better accuracy in determining the hydrometeor phase. In practice, however, the true radar reflecitvities are not always available before the phase states of the hydrometeors are determined. Therefore, it is desirable to make use of the measured radar reflectivities in classifying the phase states. To do this, a phase-identification procedure is proposed that uses only measured radar reflectivities. The procedure is then tested using APR-2 airborne radar data. Analysis of the classification results in stratiform rain indicates that the regions of snow, mixed-phase and rain derived from the phase-identification algorithm coincide reasonably well with those determined from the measured radar reflectivities and linear depolarization ratio (LDR).
\end{abstract}

Keywords: Radar, rain and snow 


\section{INTRODUCTION}

One of the important goals of the Dual-frequency Precipitation Radar (DPR) aboard the Global Precipitation Measurement (GPM) satellite is to map precipitation globally [1][2]. The DPR, operating at $\mathrm{Ku}$ - and $\mathrm{Ka}$-bands with the frequencies of 13.6 and $35.6 \mathrm{GHz}$, respectively, provides 3-dimensional measurements of precipitating hydrometeors. One of the challenges for the DPR algorithms in accurate estimates of precipitation rate is to identify hydrometeor types. Light rain exhibits a similar range of reflectivities as snow, leading to errors in separating snow, rain and mixed-phased hydrometeors from single-frequency radar measurements. The capability to distinguish hydrometeor types is important not only in achieving an accurate precipitation rate, since estimates of precipitation rate and water content differ for the cases of snow and rain, but also for weather forecasting, hydrology, detection of aviation hazards and other remote sensing applications. Moreover, the separation among regions of snow, rain and mixed phase precipitation is important in determining how to allocate estimates of total path attenuation as derived either by the radiometer or by the use of the radar surface reference technique. To explore the capability of GPM DPR for separation of snow and rain, a study by Liao and Meneghini [3] was carried out based on theoretical simulations of radar signatures in snow and rain under the assumptions of that snow follows the Gunn-Marshall size distribution [4] and rain obeys the Marshall-Palmer size distribution [5]. The study indicated that the differential frequency ratio (DFR), which is defined as the difference of radar reflectivity factors between $\mathrm{Ku}$ and Ka bands, provides useful information to distinguish snow and rain. However, the capability of separating liquid, frozen and mixed-phase hydrometeors still remains a challenge in convective rain where a clearly defined bright band is usually absent. 
The present study is aimed at the development of a dual-wavelength radar phase identification algorithm based on the principle described in [3], and examines its feasibility by applying the algorithm to the measurements taken by the Jet Propulsion Laboratory, California Institute of Technology, Airborne Precipitation Radar Second Generation (APR-2) [6] during the 2003 Wakasa Bay field campaign and the 2010 Genesis and Rapid Intensification Processes (GRIP) field experiment [7].

The linear depolarization ratio (LDR) measured by the APR-2 Ku-band provides a good indication of the mixed-phase (melting) region [8-12]. This information is used to identify regions of snow, mixed-phase and rain in stratiform storms and is taken as the true classification. On the other hand, as the DPR does not measure LDR, the idea is to link the DPR measurements of DFR and Ku-band radar reflectivity $\left(\mathrm{Z}_{\mathrm{Ku}}\right)$ on one hand with the phase states as determined from the LDR on the other. This is accomplished by the use of a look-up table that statistically links the radar parameters (DFR and $Z_{\mathrm{Ku}}$ ) of the DPR to the phase states of the hydrometeors as determined from the LDR.

It is worth noting that the fundamental difference of the present work from previous studies described by Awaka et al. [13] and Le and Chandrasekar [14] is that the objective of the former is to detect the phase states of hydrometeors while the purpose of the latter is to develop a precipitation classifier (i.e., stratiform, convective and other cases) based on features of single wavelength and dual-wavelength radar reflectivity profiles near the $0^{\circ} \mathrm{C}$ level and their horizontal gradients [13-14]. In this paper, a description of the dual-wavelength radar approach is provided 
in Section II. Analysis of the results derived from the phase identification technique is discussed in Section III followed by the remarks given in Section IV.

\section{Dual-wavelength Radar Approach}

As indicated in [3], an appropriate use of the Ku-band reflectivities and DFR of the Ku- and Kabands can form the basis for identifying the predominant phase states of hydrometeors within the storm. One way to develop a phase identification algorithm for the DPR is to construct a phase look-up table that provides the hydrometeor phase based on the values of $Z_{\mathrm{Ku}}$ and DFR, where, as noted above, the 'true' hydrometeor classification is determined from the LDR data. This look-up table can be derived through use of the results of the 2-dimensional probability density functions (PDF) for snow, rain and mixed-phase particles in the $\mathrm{Z}_{\mathrm{Ku}}-\mathrm{DFR}$ plane in which the identification of the regions of snow, rain and mixed-phase are determined from the APR-2 LDR signatures. At any point within the $Z_{\mathrm{Ku}}-\mathrm{DFR}$ plane, the phase is chosen to be that for which the PDF is greatest. For example, the snow phase is selected for a given $Z_{\mathrm{Ku}}$ and DFR if the PDF of snow is greater than those of rain and mixed phase at this location.

The LDR, which requires measurements of orthogonally-polarized returns of a polarized transmitted radar wave, is relatively weak as compared with co-polarized radar returns. The threshold used for the APR-2 data is $-30 \mathrm{~dB}$. Existence of LDR (greater than $-30 \mathrm{~dB}$ ) is presumably associated with the mixed-phase hydrometeors or melting layer. In stratiform storms, snow corresponds to the region above the mixed-phase while rain is below the melting layer. Although Ku-band radar reflectivity measurements show distinct profiles within the melting region with enhanced echoes (namely bright- 
band), a difficulty usually arises in determining the position where the melting starts in a vertical profile. In typical Ku-band radar vertical profiles of stratiform rain, the radar reflectivities gradually increase prior to the melting because of aggregations, leading to ambiguity in finding the starting point of melting. Le and Chandrasekar [14] attempt to link the maximum of the DFR gradient to the location where the melting begins. Because of the somewhat noisy DFR gradients along the range direction, a smoothing scheme is needed. The Ka-band radar bright-band signature, on the other hand, shows a smaller, less distinct bright-band signature than its Ku-band counterpart. In view of various reflectivity measurements, the LDR signature in the melting region appears to be not only the most distinctive but also the most robust. As a result of this, the LDR data are exclusively used in our study to identify mixed-phase regions.

Fig.1 depicts results from the look-up table obtained from the APR-2 data collected on 23 January 2003 in stratiform storms during the Wakasa Bay field campaign. The data employed in generating the table include all of the measurements of the APR-2 (from 23 equally spaced angle bins), covering a cross-track scan $\pm 25^{0}$ from nadir. Shown in Fig. 2 is a segment of the APR-2 measurements at nadir on 23 January 2003 in stratiform rain, in which the Ku- and Ka-band radar reflectivity factors are given in the top and middle panels while the LDR is displayed in the bottom panel. As reference, theoretical relations between DFR and $Z_{\mathrm{Ku}}$ for snow (thin curves) are plotted in Fig.1 assuming a constant snow density $\left(\rho_{\mathrm{s}}\right)$ and the Gunn-Marshall size distribution [4]. Similarly, the DFR- $Z_{\mathrm{Ku}}$ relationship for rain is given by the heavy-solid line using the Marshall-Palmer raindrop size distribution [5]. Curves of constant rain rate (R) are shown by the thin solid curves. In generating the phase look-up table, attenuation-corrected DFR and Ku-band radar reflectivity factors are adopted. For obtaining attenuation corrected reflectivities, the 
surface reference technique (SRT) is incorporated into the dual-wavelength radar retrieval algorithms [15-18], which is then applied to the APR-2 data. It is worthwhile noting that attenuation due to rain and mixed-phase particles may complicate the identification because Kaband undergoes much more attenuation than the Ku-band, resulting in an increase in the DFR. As will be discussed later in the paper, failure to correct for attenuation can lead to misclassification of the hydrometeors.

As described above, the attenuation-corrected reflectivities are required for the look-up table. In the operational radar algorithms, the hydrometeor phases, however, need to be known before performing the attenuation correction procedure that, in fact, depends on phase classification. An iterative procedure is a possible way to derive the hydrometeor phases and attenuation but such a procedure is computationally intensive and complex. An alternative method is to make use of the characteristics of precipitation structures, using, for example, the fact that snow is unlikely to be present below rain and mixed-phase regions.

\section{Results}

As a first check, we apply the phase-state look-up table directly to the APR-2 radar data without attenuation correction. Fig.3 (a-d) provides an example of the APR-2 Ku- and Ka-band radar measurements over a stratiform storm in which a clear radar bright-band is detected. The melting layer is also clearly indicated by the Ku-band LDR signatures (Fig.3d). Using the measured Ku-band reflectivities and DFR of Fig.3a and 3c as the inputs of the look-up table of Fig.1, the hydrometeor phases are determined. The results are shown in Fig.3e. Since snow attenuation is generally 
negligibly small at both $\mathrm{Ku}$ - and Ka-bands, there is a good agreement between the snow regions retrieved from the phase look-up table using the measured reflectivities (blue area of Fig.3e) and those known from the stratiform vertical profiles (in which snow is exclusively present above the mixed-phase region). In other words, for the purpose of snow identification, sufficient accuracy is obtained by using the measured radar reflectivities. As also can be seen in Fig.3e, the melting layer (red) and most of the rain (yellow) inferred from the measured reflectivities appear in good agreement with the radar reflectivity and LDR measurements depicted in Fig.3a-d. Some of the rain regions, however, are misclassified as mixed-phase largely due to the difference in attenuation between the $\mathrm{Ku}-$ and Ka-band data accumulated through the melting layer and rain. Because of the cumulative effects of attenuation, misclassification of the phase state usually occurs in the regions near the surface and in locations where the attenuations are severe. Fig.3f provides information on agreement of the identified phase states shown in Fig.3e with those derived from the LDR signatures. The pixels in green indicate agreement while the black represents disagreement. The results show that the areas where the DFR-and LDR-based results frequently disagree occur near the boundary of snow and mixed-phase and boundary of rain and mixed-phase. Unlike the LDR signature, which has an abrupt beginning and end, the DFR and Ku-band reflectivities vary more gradually over these transition regions so that a clear demarcation of the mixed phase region is more difficult to determine. It is worth noting that the LDR, on the other hand, is not "perfect" because of its limited sensitivity in measurements and insensitivity to nearly spherical particles. It therefore results in some possible uncertainties in phase identifications using LDR near the boundaries between snow and mixed-phase as well as between mixed-phase and rain. 
Because the hydrometeor phases of the stratiform storm are clearly defined just from the radar returns and also because the APR-2 measurements are made over the ocean (as shown from steady and strong surface returns), the attenuations can be corrected by using the SRT and the dual-wavelength backward techniques [15-18]. With the attenuation-corrected reflectivities the hydrometeor phases are derived and depicted in Fig.3g. It is evident that there is an overall improvement in phase identification if the attenuation-corrected radar reflectivities are used instead of the measured ones (no attenuation correction) despite the fact that a very small portion of the rain data below and adjacent to the strong bright-band are still misclassified as the mixedphase. This is also indicated in Fig.3h, in which the locations of the agreement/disagreement between the phase state results derived from the LDR measurements and those inferred from the attenuation-corrected DFR- $Z_{\mathrm{Ku}}$ technique. Although the accuracy of hydrometeor phase identification could be improved using attenuation-corrected reflectivities, the attenuations are difficult to correct accurately without knowing the phase states of hydrometeors along the radar path. It is therefore desirable from the perspective of the radar algorithms to classify the hydrometeor phases using the measured radar reflectivities. What follows is a discussion on a possible means of improving the accuracy of phase classifications by taking into account some of the precipitation features.

As seen in Fig.1, rain is generally associated with relatively small values of DFR and a broad range of possible Ku-band reflectivities. Precipitating hydrometeors result in more severe attenuation at Ka-band than at Ku-band, leading to an increase of DFR if there is no attenuation compensation. An enhanced DFR, in turn, can lead to rain misidentified as snow for small $Z_{\mathrm{Ku}}$ and mixed phase for large $Z_{\mathrm{Ku}}$ in accordance with the phase look-up table. Generally, attenuation 
doesn't significantly impact the results of snow and mixed-phase classifications in part because separation of snow and mixed-phase is primarily determined by the values of $Z_{\mathrm{Ku}}$. The $Z_{\mathrm{Ku}}$ is less attenuated over a short path within the melting layer and experiences almost no attenuation in the snow region. An increase of DFR resulting from Ku- and Ka-band attenuations actually improves the identification of snow and mixed phase for a given value of $Z_{\mathrm{Ku}}$ because it forces the data away from the region of rain in the $Z_{\mathrm{Ku}}$-DFR plane. As illustrated in Fig.3f, when attenuation is not taken into account, the most common error occurs when rain is misclassified as either mixed-phase or snow. This usually happens when the PIA is large, such as near the surface and in regions of intense precipitation. In view of the fact that snow is unlikely to be present below the rain and mixed-phase regions and that mixed phase doesn't often appear below the rain, it is possible to improve the accuracy of phase identification if these storm features are considered.

Using the measured reflectivities and taking into account the precipitation features described above, the phase states of the hydrometeors are identified by the following procedure. For airborne or spaceborne radars, the phase state is determined starting from the storm top, and then moving downward until reaching the earth surface. Cases where the LUT classifies the hydrometeors just below rain field/column as snow or mixed-phase are considered incorrect and changed to the rain category. Likewise, if mixed phase, as determined by the LUT, is found below the rain field/column, the classification is changed to rain. For the APR-2 data, a rain field/column is defined as a range profile consisting of at least 20 consecutive rain range gates. 
Fig.4 provides examples of hydrometeor phases (Fig.4e) identified by the APR-2 Ku- and Kaband measured radar reflectivities using the phase look-up table of Fig. 1, aided by the use of precipitation features described previously. Note that in using the LUT, the measured $\mathrm{Z}_{\mathrm{Ku}}$ and DFR are taken to be the attenuation-corrected values. Also note that in this classification, the LDR is not used but is considered as the true location of the mixed-phase region.

While the radar data in these two examples are taken from measurements during the Genesis and Rapid Intensification Processes (GRIP) experiment on 1 September (left column) and 30 August (right column) of 2010, the phase look-up table adopted for the phase determination is the one shown in Fig.1, derived from the radar data from a different field campaign (2003 Wakasa Bay). It is not difficult to see from the left column of Fig.4 (September case) that the mixed-phase region (bright-band) inferred from the APR-2 Ku- and Ka-band measured reflectivity factors coincides fairly well with that detected by the Ku-band LDR. Most of the snow above and the rain below the bright band are correctly identified. An obvious improvement in the classified phases shown in Fig.4d-e, as compared with those in Fig.3e-f, is a significant reduction in the number of rain misclassifications. This can be further viewed from the comparisons of Tables 1 and 2, in which the percentages of the total data points that are classified as one phase state (e.g., snow, rain or mixed phase) from the $\mathrm{Z}_{\mathrm{Ku}}$-DFR relations to the phase states determined by the $\mathrm{Ku}$ band LDR signatures. The data used in compiling the table are exclusively from the stratiform events during GRIP in which the LDR measurements are able to precisely separate the snow, rain and mixed-phase regions and thereby provide reliable spatial information on the hydrometeor phase state. The differences between Tables 1 and 2 arise from the fact that the former uses the measured reflctivities alone for phase identification while the latter makes use of 
both the measured reflectivities and the precipitation features. The diagonal values of the tables depict percentages of agreement of the phase states as estimated by the $\mathrm{Z}_{\mathrm{Ku}}-\mathrm{DFR}$ and by the LDR signatures while the off-diagonal values show the percentages of the data that are misclassified. Clearly, the accuracy of rain identification is greatly improved if precipitation features are taken into account in that there is an increase in agreement from $50 \%$ to $94 \%$ in rain classification before and after using precipitation features. In general, there is a good accuracy $(92 \%$ agreement) for snow classification. However, only $63 \%$ of the mixed-phase area, which primarily corresponds to the region where the bright-band appears, is correctly classified. Of the $37 \%$ of data misclassified, $11 \%$ and $26 \%$ of the mixed-phase area are misidentified as snow and rain, respectively. An improvement in the identification of the mixed-phase might be achieved by modifying the LUT by using a larger database containing mixed-phase measurements. Despite this deficiency, in general, fairly good agreement between the results of the hydrometeor phase regions derived through the look-up table and the results from the LDR suggests that the dual-wavelength techniques based exclusively on the measured $\mathrm{Ku}$ - and Ka-band radar reflectivities are effective when taking into account some of the precipitation features. The validity of the phase look-up table generated from one field campaign and applied successfully to radar data from a different field campaign shows the consistency of the microphysical properties of snow, mixed-phase and rain hydrometeors and the associated radar measurements.

Shown in the right column of Fig. 4 are measurements made on 30 August 2010 over stratiform rain with several embedded convective structures. The $Z_{\mathrm{Ku}}$-DFR classification results identify liquid water rising above the $0^{0} \mathrm{C}$ level during the early and middle measurement period. This, however, contradicts results obtained from the LDR classification, shown in the dark area of the bottom panel 
of the right column in Fig.4. The APR-2 Ku-band reflectivity-weighed mean Doppler velocity (not shown) indicates that there exist moderate updrafts associated with these areas. Because of this and also because of the absence of an LDR signature, one can argue that it is possible that in these areas liquid water (or nearly spherical wet graupel) and snow coexist. The presence of liquid water or wet graupel (usually with a mass density greater $0.4 \mathrm{~g} / \mathrm{cm}^{3}$ ) leads to a reduced DFR and enhanced Kuband reflectivity, and as a result, pushes the classification toward the rain category. Accurate validation of this, however, is hard to achieve because of the lack of direct independent measurements. The LDR signatures, though useful in identifying regions of mixed-phase, are not effective in detecting liquid water above the $0{ }^{\circ} \mathrm{C}$ level.

Once rain columns/fields are identified, all precipitation below this is assigned to the rain category according to the precipitation features used in the DFR algorithm. Because of this, the DFR algorithm misclassifies some of the mixed-phase regions, which are clearly identified by the LDR signatures. This constitutes one of the weaknesses of the algorithm as applied to convective rain. Another possible deficiency in applying the phase look-up table of Fig.1 (built exclusively from the stratiform rain) to the convective cases arises from the fact that the look-up table doesn't include data from dry or melting graupel that are often present in convective rain. To improve the efficiency of convective phase classification, it would be necessary to include radar data measured directly from convective rain in constructing either a more complete phase look-up table or an alternative table exclusively for convection. To achieve this, knowledge of the phase states will be required. Measurements from dual-wavelength full-polarimetric and Doppler radar might be useful for this purpose [10]. 


\section{Remarks}

Determination of hydrometeor phase states is an important element in developing an accurate GPM DPR algorithm to estimate the precipitation rate. This study is aimed at investigating the feasibility of a dual-wavelength radar algorithm to identify and discriminate the various phase states of precipitating hydrometeors. In the study, a dual-wavelength radar phase algorithm is developed from the radar measurements of snow, mixed-phase and rain obtained from airborne data with measurements of LDR. The algorithm, presented in the form of a look-up table that links the Ku-band radar reflectivities and DFR directly to the phase states of hydrometeors, is checked by employing the APR-2 data taken from the measurements during the 2003 Wakasa Bay and the 2010 GRIP field campaigns. For validation purposes, most of the tests are made for the case of stratiform storms as their phase states can be fairly accurately determined with aid of the LDR signatures that clearly define the melting layer or mixed-phase region that separate the snow and rain regions. As the phase look-up table is formed by using the attenuation-corrected $\mathrm{Ku}-$ and $\mathrm{Ka}$-band radar reflectivities, the true radar reflectivity factors are required for the phase determination. However, as attenuation-correction algorithms require knowledge of the hydrometeor phase states along the path, the attenuation-corrected reflectivities are not available for use in the classification procedure unless an iterative procedure is employed. One of the drawbacks of using measured reflecitvities is the increased probability of misclassifying rain as either mixed phase or snow because of the enhanced DFR caused by the differential Ku/Ka-band attenuation. It is also worth mentioning that the applicability of the dual-wavelength technique is limited to cases where the Ka-band signal is detectable. Because of attenuation, the DFR is not 
always available. This is one of the additional drawbacks in classifying the hydrometeor phase state using DFR data.

To circumvent the need for using true radar reflectivities in determining hydrometeor phase, the measured radar reflectivities and look-up table are used along with some precipitation features for estimating phase. Results from this study indicate that regions of snow, mixed-phase and rain derived from the measured $\mathrm{Ku}$ - and Ka-band radar reflectivities, having taken into account precipitation features, agree reasonably well with those obtained from the LDR for stratiform events. While the dual-wavelength phase algorithm is useful to identify precipitation phase (rain or snow), another important issue for future studies is to determine whether a technique that is trained on radar measurements of stratiform rain, is applicable to convective cases in which bright band signatures are absent.

\section{ACKNOWLEDGEMENTS}

The authors wish to thank Dr. Eastwood Im and Dr. Simone Tanelli of the NASA Jet Propulsion Laboratory for providing the APR-2 radar data and the processing software. This work is supported by Dr. R. Kakar of NASA Headquarters under NASA's Precipitation Measurement Mission (PMM) GrantNNH12ZDA001N-PMM. 


\section{References}

[1] A. Hou, G. S. Jackson, C. Kummerow, and C. M. Shepherd, 2008,"Global precipitation measurement. Precipitation: Advances in Measurement, Estimation, and Prediction, S. Michaelides,'Ed., Springer, pp. 131-169, 2008.

[2] Hou, A. Y., R. K. Kakar, S. Neeck, A. A. Azarbarzin, C. D. Kummerow, M. Kojima, R. Oki, K. Nakamura, and T. Iguchi, "The global precipitation measurement mission," Bull. American Meteor. Soc., vol. 95, pp. 701-722, 2014.

[3] L. Liao and R. Meneghini, "A Study on the feasibility of dual-wavelength radar for identification of hydrometeor phases," J. Appl. Meteorol. Climatol., vol. 50, pp. 449-456, 2011.

[4] K. L. S. Gunn and J.S. Marshall, "The distribution with size of aggregate snowflakes," J. Meteor., vol. 15, pp. 452-461, 1958.

[5] J. S. Marshall and W.M. Palmer, "The distribution of raindrops with size," J. Appl. Meteor., vol. 5, pp. 165-166, 1948.

[6] G. A. Sadowy, A. C. Berkun, W. Chun, E. Im, and S. L. Durden, "Development of an advanced airborne precipitation radar,"Microwave Journal, vol 46, pp. 84-98, 2003.

[7] E. Im, “APR-2 dual-frequency airborne radar observations, Wakasa Bay, Boulder, CO: National snow and ice data center," Digital media, 2003.

[8] H. Liu, and Chandrasekar, V., "Classification of hydrometeors based on polarimetric radar measurements: Development of fuzzy logic and neuro-fuzzy systems, and in situ verification,” J. Atmos. and Oceanic Technol, vol. 17, pp. 140-164, 2000.

[9] D. Zrnic, Ryzhkov, A., Straka, J., Liu Y., Vivekanandan, J., "Testing a procedure for automatic classification of yydrometeor types," J. Atmos. and Oceanic Technol., vol. 18, pp. 892-913, 2001.

[10] S. Tanelli, J. P. Meagher, S. L. Durden and E. Im, "Processing of high resolution, multiparametric radar data for the Airborne Dual-Frequency Precipitation Radar APR-2," SPIE Fourth Int'l Asia-Pacific Symposium on Remote Sensing, Honolulu, HA, Nov 2004. SPIE 5654, pp. 25-32, 2004.

[11] B. Reinhart, H. Fuelberg, R. Blakeslee, D. Mach, A. Heymsfield, A. Bansemer, S. L. Durden, S. Tanelli, G. Heymsfield, B. Lambrigtsen, "Understanding the Relationships between Lightning, Cloud Microphysics, and Airborne Radar-Derived Storm Structure during Hurricane Karl," Monthly Weather Review, vol. 142, pp. 590-605, 2010. doi: http://dx.doi.org/10.1175/MWR-D-13-00008.1 
[12] R. Bechini and V. Chandrasekar, "A semisupervised robust hydrometeor classification method for dual-polarization radar applications," J. Atmos. Oceanic Technol., vol. 32, pp. $22-47,2015$.

[13] J. Awaka, T. Iguchi, and K. Okamoto, "TRMM PR standard algorithm 2A23 and its performance on bright-band detection,” J. Meteor. Soc. Japan, vol. 87A, pp. 31-52, 2009.

[14] M. Le and V. Chandrasekar, "Precipitation type classification method for dual-frequency precipitation radar (DPR) onboard the GPM," IEEE Trans. Geosci. Remote Sens., vol. 51, pp. 1784-1790, 2013.

[15] R. Meneghini, T. Kozu, H. Kumagai, and W. C. Boncyk, "A study of rain estimation methods from space using dual-wavelength radar measurements at near-nadir incidence over ocean," J. Atmos. Oceanic Technol., vol. 9, pp. 364-382, 1992.

[16] R. Meneghini, T. Iguchi, T. Kozu, L. Liao, K. Okamoto, J.A. Jones and J. Kwiatkowski, "Use of the surface reference technique for path attenuation estimates from the TRMM radar," J. Applied Meteorology, vol. 39, pp. 2053-2070, 2000.

[17] L. Liao and R. Meneghini, "A study of air/space-borne dual-wavelength radar for estimates of rain profiles," Advances in Atmos. Sci., vol. 22, pp. 841-851, 2005.

[18] L. Liao, R. Meneghini, L. Tian, and G. M. Heymsfield, "Retrieval of snow and rain from combined X- and W-band airborne radar measurements," IEEE Trans. Geosci. Remote Sens., vol. 46, pp. 1514-1524, 2008. 
Table 1: Percentages of the estimated phases (denoted by 'E. Snow', 'E. Rain' and 'E. Mixed') obtained from the $Z_{\mathrm{Ku}}$-DFR LUT using measured reflectivities to the 'true' values obtained by means of the LDR for stratiform events.

\begin{tabular}{|l||c|c|c|}
\hline \multicolumn{1}{|c||}{} & \multicolumn{3}{c|}{ Measured Z } \\
\cline { 2 - 4 } & E. Snow & E. Rain & E. Mixed \\
\hline Snow & $92 \%$ & $18 \%$ & $11 \%$ \\
\hline Rain & $0 \%$ & $50 \%$ & $26 \%$ \\
\hline Mixed & $8 \%$ & $32 \%$ & $63 \%$ \\
\hline
\end{tabular}

Table 2: Same as Table 1 but using both the measured $\mathrm{Z}_{\mathrm{Ku}}$-DFR and some precipitation features for the hydrometeor phase identification.

\begin{tabular}{|l||c|c|c|}
\hline \multicolumn{1}{|c||}{} & \multicolumn{3}{c|}{ Measured Z \& Precip. Features } \\
\cline { 2 - 4 } & E. Snow & E. Rain & E. Mixed \\
\hline Snow & $92 \%$ & $3 \%$ & $11 \%$ \\
\hline Rain & $0 \%$ & $94 \%$ & $26 \%$ \\
\hline Mixed & $8 \%$ & $3 \%$ & $63 \%$ \\
\hline
\end{tabular}


Figure captions

Fig.1 Phase map of hydrometeors developed from the APR-2 measurements from stratiform rain events taken on 23 January 2003 . For any point in the DFR-Z $Z_{\mathrm{Ku}}$ plane, the phase state is taken to be that for which the corresponding PDF is largest.

Fig.2 Measurements of the radar reflectivity factors at Ku-band (top) and Ka-band (middle) and LDR (bottom) at Ku-band taken from the APR-2 during the Wakasa Bay AMSR-E field campaign on 23 January 2003.

Fig.3 Examination of phase identification scheme using the phase map shown in Fig.1 for the APR-2 stratiform measurements at nadir on 23 January 2003. (a): Ku-band reflectivity. (b): Kaband reflectivity. (c): Ku- and Ka-band differential frequency ratio (DFR). (d): LDR at Ku-band (indication of melting region). (e): Phase states determined from $\mathrm{Ku}$ - and $\mathrm{Ka}$-band measured (w/o attenuation correction) radar reflectivities. (f): Agreement/disagreement of the identified phase states in Fig.3e with the LDR-based results. (g): Phase states determined from Ku- and Kaband attenuation-corrected radar reflectivities. (h): Agreement/disagreement of the identified phase states in Fig.3g with the LDR-based results.

Fig.4 Hydrometeor phase classification ( $2^{\text {nd }}$ from the bottom, panel-d) determined from APR-2 measured radar reflectivities (top 2 panels, a \& b) and a look-up table (Fig.1) with the aid of some precipitation features. The data are taken from the APR-2 GRIP experiment in 2010. Agreement/disagreement of the identified phase states with the LDR-based results are given in the bottom panels (e), in which the pixels with green color indicate an agreement while those in black represent that two results disagree. 


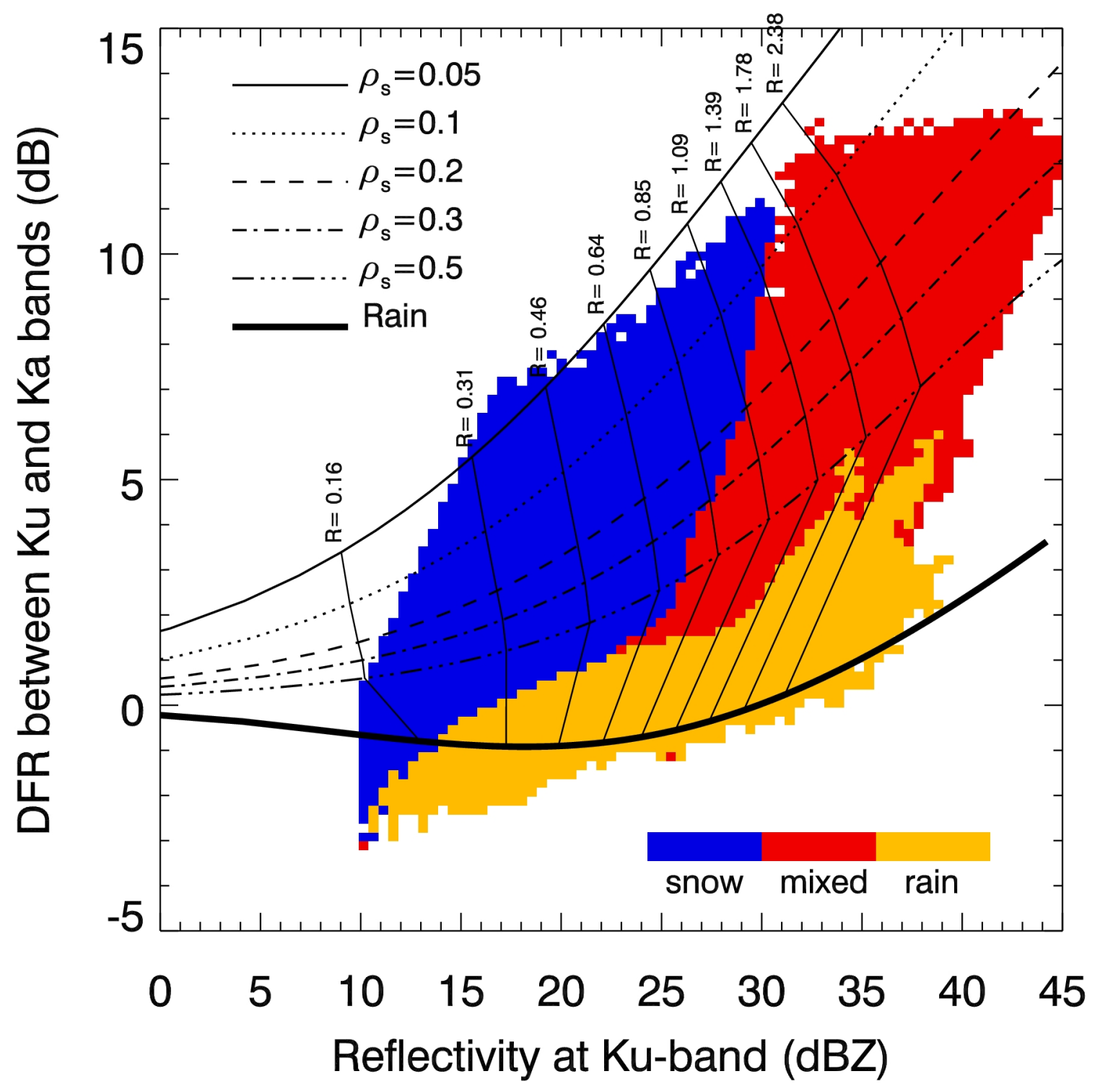

Fig.1 Phase map of hydrometeors developed from the APR-2 measurements from stratiform rain events taken on 23 January 2003. For any point in the DFR- $Z_{\mathrm{Ku}}$ plane, the phase state is taken to be that for which the corresponding PDF is largest. 


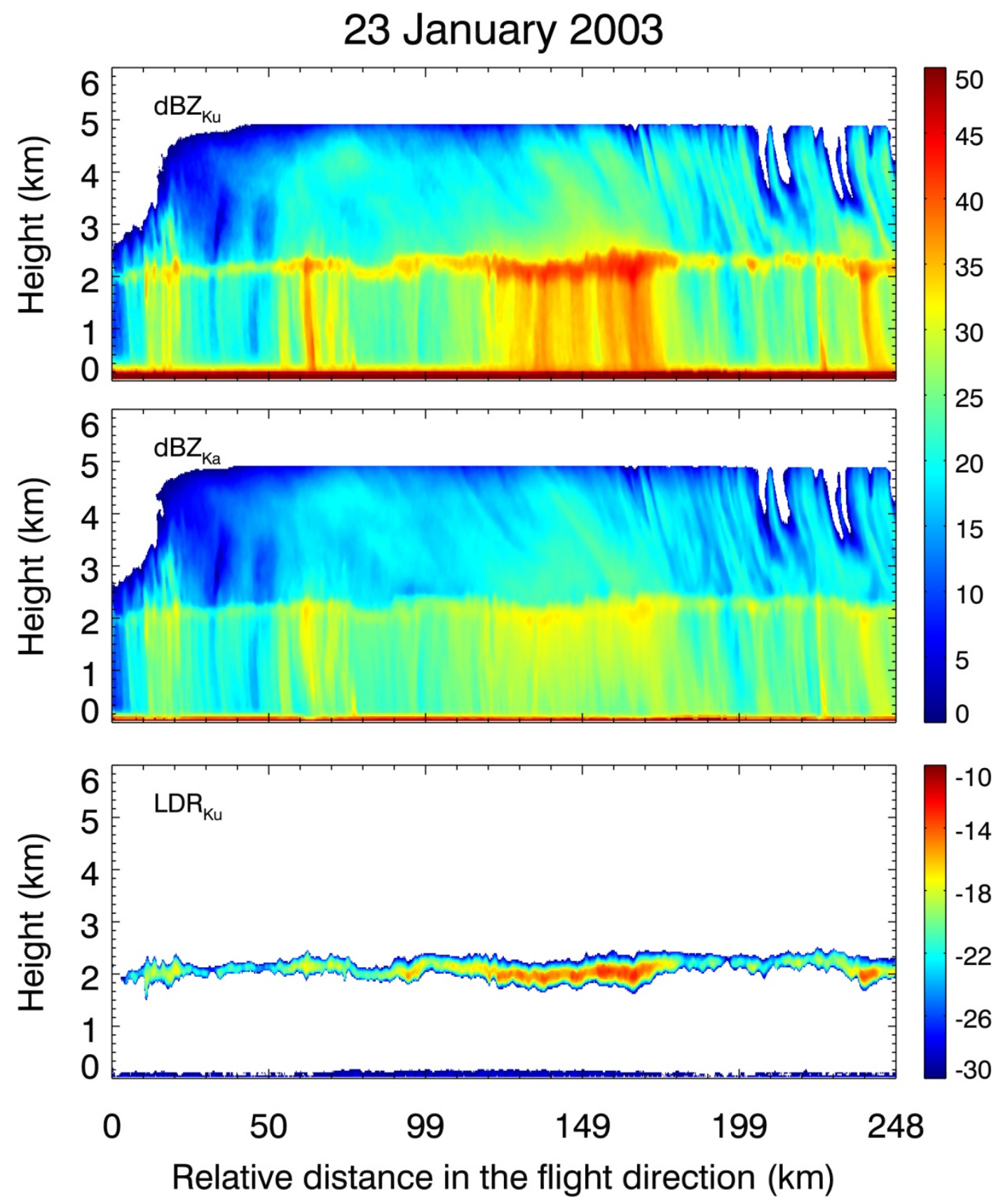

Fig.2 Measurements of the radar reflectivity factors at Ku-band (top) and Ka-band (middle) and LDR (bottom) at Ku-band taken from the APR-2 during the Wakasa Bay AMSR-E field campaign on 23 January 2003. 

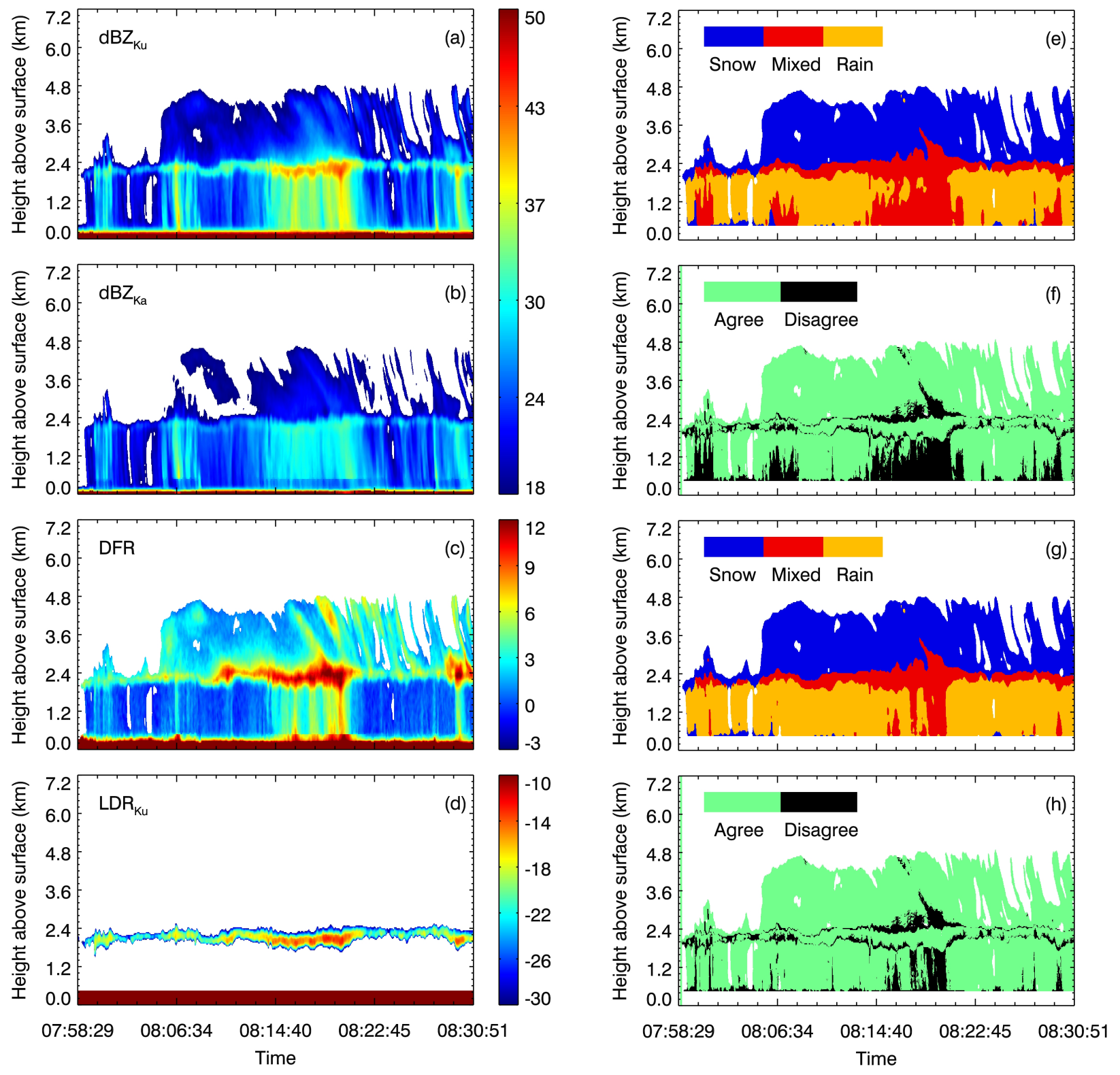

Fig.3 Examination of phase identification scheme using the phase map shown in Fig.1 for the APR-2 stratiform measurements at nadir on 23 January 2003. (a): Ku-band reflectivity. (b): Kaband reflectivity. (c): Ku- and Ka-band differential frequency ratio (DFR). (d): LDR at Ku-band (indication of melting region). (e): Phase states determined from $\mathrm{Ku}$ - and Ka-band measured (w/o attenuation correction) radar reflectivities. (f): Agreement/disagreement of the identified phase states in Fig.3e with the LDR-based results. (g): Phase states determined from Ku- and Kaband attenuation-corrected radar reflectivities. (h): Agreement/disagreement of the identified phase states in Fig.3g with the LDR-based results. 

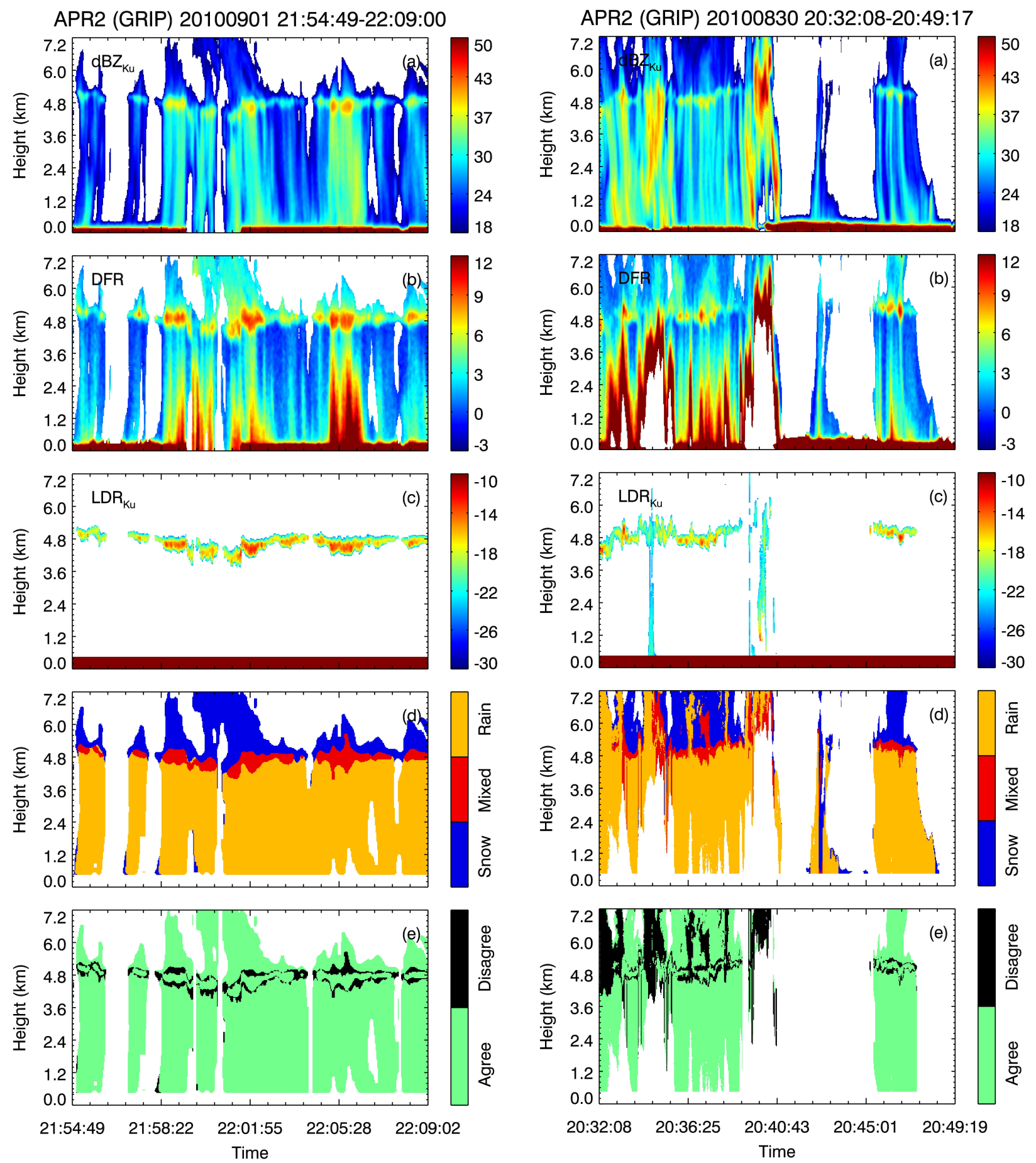

Fig.4 Hydrometeor phase classification ( $2^{\text {nd }}$ from the bottom, panel-d) determined from APR-2 measured radar reflectivities (top 2 panels, a \& b) and a look-up table (Fig.1) with the aid of some precipitation features. The data are taken from the APR-2 GRIP experiment in 2010. Agreement/disagreement of the identified phase states with the LDR-based results are given in the bottom panels (e), in which the pixels with green color indicate an agreement while those in black represent that two types of the results disagree. 